\title{
$\mathfrak{S} \mathfrak{i} \mathfrak{a} \mathfrak{f} \mathfrak{u} \mathfrak{t}$
}

Dea

\section{Deutiden Gurifentages.}

$\S 1$.

Der 3med des beutichen Suriftentages iit: eine Bereinigung für ben lebendigen Meinungs̄austaufd und ben perfönlifjen Berfehr unter ben beutiden Suriften zu bilben; auf ben Bebieten bes \$rivatredts, bes Proceffes und bes Strafredts Den Frorberungen nad einheitlidjer Ent= wiffelung immer gröpere 2 nerfennung zu verfdaffen, bie Şinberniffe, weldye biejer Entwifelung entgegenftehen, zu bezeidgnen unb fid über $\mathfrak{B}_{0}=$

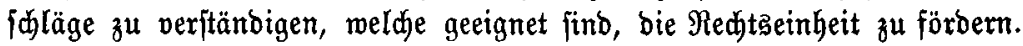

$\S 2$.

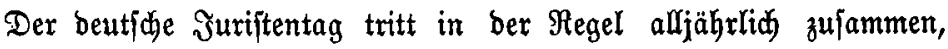
boch ijt bie ftändige Deputation ermädtigt, aus Gründen ber 3weds

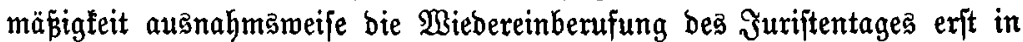
bem auf befjen leţten 3 ufammentritt folgenden zmeiten Ralenderjahre vorzunehmen.

\section{$\S 3$.}

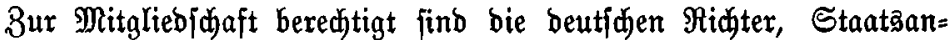

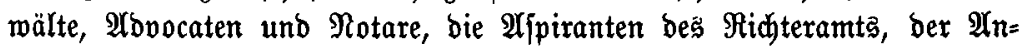
waltjdaft unb bes Rotariats, fomie jeber, ber naw jeinen Randeşgejeţen

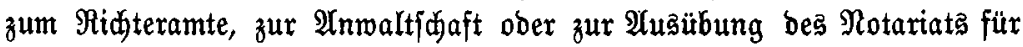
befähigt erfannt ijt, ferner bie Rehrer an ben beutiden F̧odjđdulen, bie Mitglieber ber gelehrten $\mathfrak{A c a b e m i e n , ~ b i e ~ D o c t o r e n ~ b e r ~ \Re e f f i t e ~ u n d ~ b i e ~}$ red)tägelehrten Mitglieber ber Berwaltungşbełörben. 
$\S 4$.

Die Mitgliebjøaft beginnt mit bem Empfange ber Mitgliebăfarte. Sie beredtigt zur Theilnahme an ben Berhandlungen und an ber Abjitimmung.

\section{$\S 5$.}

Der Beitrag ber Befellfdafts̄mitglieder beträgt Seç̉ Marf jährlid

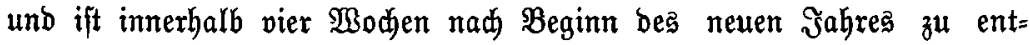
ridften, mibrigenfalls berfelbe burd \$oftworfdun eingezogen miro. Nimmt

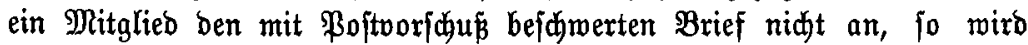

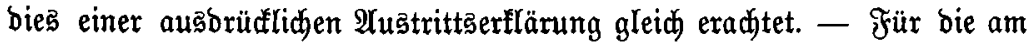

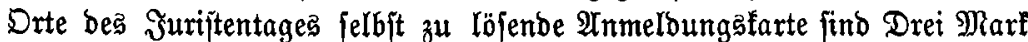
子u entrififten.

\section{$\S 6$.}

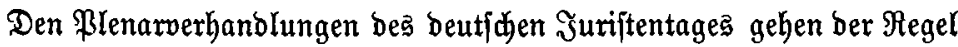

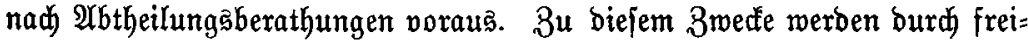
willige Finzeidnnungen ber Mitglieber folgenbe vier 2 (fththeilungen gebilldet:

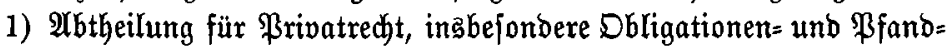
redit, juriftifdes Stubium und praftifde $\mathfrak{A}$ usbildoung.

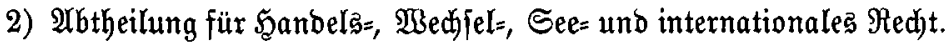

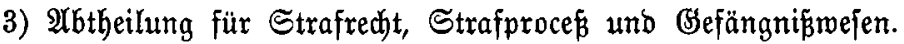

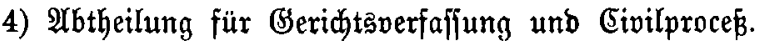

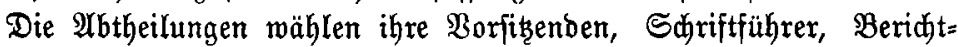
eritatter unb benadridtigen Den Borithenden ber Blenarverfammlung (§ 7), jobald ihre Berathungen über einzelne Begenjtände gefolloffen

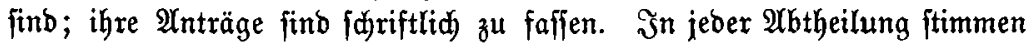
nur Diejenigen mit, weldhe fidy in bie betreffende $\mathfrak{A b t h e i l u n g}$ bereits ein= gezeidnet haben.

Sämmtlidbe Beidhlüffe ber Mbtheilungen werben in ber Plenarver= fammlung mitgetheilt. Es finbet jebod eine Erörterung und Entjujeibung im Blenum nur bann jtatt, wenn biejelbe von ber betreffenden Albthei= lung vorgejdlagen, ober wenn fie von mindejtens zehn Mitgliebern be= antragt unb von ber Blenarverfanmlung bejdlofien wirb. Uleber bie Borfrage, ob bem von minbeftenz zehn Mitgliebern gejtellten 2 nntrage

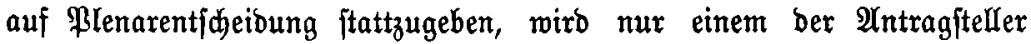
und bem Beridjterftatter bas $\mathfrak{B}_{0}$ rt ertheilt.

$\S 7$.

Die Berhandlungen ber \$lenarwerjammlung leitet ein Borfiţender, meld)er für bie Dauer eines jeben Suriftentages in ber erften Plenarver= fammlung burd, Stimmzettel ober Acclamation gemählt wirb. Derfelbe 
benennt zmei bis vier Stellvertreter und vier Sdriffführer. (5r bejtimmt bie Tageborbnung und fann einzelne Segentänoe, ohne Borberathung in

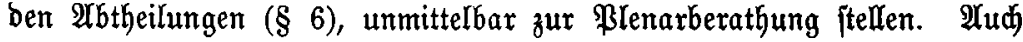
ift ex befugt, Nidhtmitglieber als 3 uhörex zuzulafjen.

$\S 8$.

Bei allen Bejdlüjjen bex ßlenarverjammlung und ber 2rbtheilungen entjąeibet einfadje Majorität ber anmejenden Mitglieber, bet allen Maglen relative Majorität uno im Falle ber Stimmengleidheit bas Soos.

\section{$\S 9$.}

Wirb Shlup ber Debatte beantragt, fo wirb über biejen Intrag fofort abgeftimmt. In ber \$lenaroerfammlung find alle $\mathfrak{A}$ nträge mit

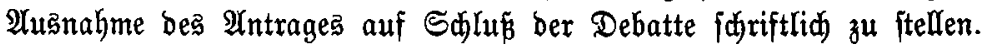

\section{0 .}

Bor bem Sdluffe eines jeben Sัuriftentages wiro von ber \$lenar= verjammlung burch acclamation ober in einem einzigen Scrutinium burd) Stimmzettel eine aus neunzehn Mitgliebern und bem ßräjíbenten deả leb̧ten Jurijtentages als Ėhrenpräjibenten beltehende ftänoige Deputa= tion gemählt. Die Sifte ber zur $\mathfrak{A}$ cclamation vorzufblagenden Berjonen wiro burd den ßräfíbenten ber ßlenarverfammlung, feine Stellvertreter und je zehn von jeber abtheilung gewählte Bertrauenämänner gemein= (d)aftlid) feftgeitellt.

Die ftändige Deputation hat folgenbe Befugniffe und Dbliegenheiten:

1) fie forgt für bie $\mathfrak{2}$ uşührung ber von bem Juriftentage gefapten Befdlüfje, veranlajat nad) eigenem Ermefien ben Drud ber ßro= tocolle und Borlagen, bewirft die Bertheilung ber Druffachen an bie Mitglieber und vermahrt alle Acten und Sdjriftitide bes Suriftentages;

2) fie beftimmt 3eit und Drt Des nädjten Juriftentagesె, trifft bie für benjelben nöthigen Borbereitungen, erläpt bie Einlabungen, ftellt bie vorläufige Tagesorbnung auf, wobei fie in ber Fegel nur bie bis̄ zum 31. Mai bes laufenden $\mathfrak{J} a \mathfrak{h r e s}$ eingegangenen

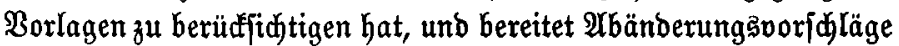
in Betreff ber Bejdäftsoronung für bie ßlenarverjammlung vor;

3) fie nimmt bie Beitritţerflänungen neuer Mitglieber entgegen, fer= tigt bie Mitgliebsłârten aus, empfängt bie Beiträge, bejtreitet

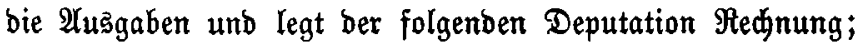


4) fie ergängt fỉ jelb̆t, falfa einz ober mehrere Mitglieber während

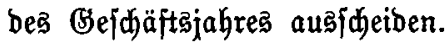

Die Deputation wäblt aus ihrer Mitte einen Borfitzenben, einen

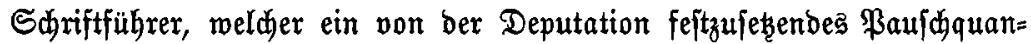
tum für baare 2luslagen erbält, und einen Baffirer. Der Seţtere ift ver= pflidftet, Der jtändigen Deputation bei ifrem jebesmmaligen $3 \mathfrak{u}\{\mathfrak{a m m e n t r i t t}$

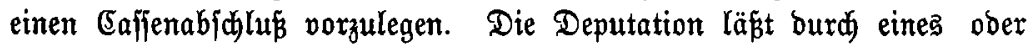
mehrere ihrer Mitglieber bie Rednung prïfen und bie $\mathfrak{C}_{a f f e}$ revidiren.

Die Deputation bejtimmt Drt und Beit ihrex 3ufammenfunft. Zur (bültigfeit ihrer $\mathfrak{B}$ efd̆lüfje ift bie Einladung jämmtlider Mitglieber, fowie bie Mitmirfung von menigftens fünf Mitgliebern erforberlid.

\section{$\S 11$.}

2rbänberungen biejes Statuts fönnen zwar von ber Plenarverjamm=

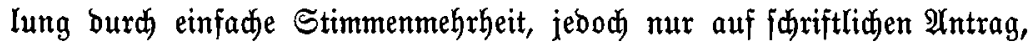

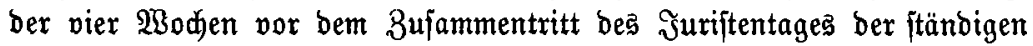
Deputation (\$ 10) überreidjt worben, befdhloffen werben. 